Revista Ciencias Sociales y Económicas - UTEQ (2018)

ISSN 2588-0586 IMPRESO; ISSN 2588-0594 ELECTRÓNICO

Volumen 2, Número 1. Semestral (enero-junio)

\title{
La Seguridad del Transporte Terrestre: Retos y Perspectivas
}

\section{The Safety of Land Transport: Challenges and Perspective}

\author{
Sara Jeaneth Malave Drouet \\ Universidad de las Fuerzas Armadas ESPE - Ecuador \\ sjmalave@espe.edu.ec
}

Fecha de recepción: 09/01/2018

Fecha de aceptación: 01/04/2018

Publicado: 29/06/2018

\begin{abstract}
Resumen
El transporte terrestre es aquel traslado que se realiza sobre la superficie de la tierra, la gran mayoría se realizan sobre ruedas, tiene su origen en el traslado del ser humano, quien por la necesidad de moverse o de trasladar sus cargas atendiendo al peso o la distancia se vio en la necesidad de perfeccionar este mecanismo, desde el empleo de los ríos hasta la tierra firme. El devenir del tiempo provocó la aparición de la rueda en lo sucesivo del motor, que también se vio inmerso en su evolución y perfeccionamiento, primero empleó el fuego, el agua, el vapor y luego aparece el motor de combustión interna, perfeccionándose con el descubrimiento del petróleo. En la actualidad el transporte terrestre apunta hacia nuevas alternativas como la electricidad, la energía solar y la inteligencia artificial.
\end{abstract}

Palabras Clave: Seguridad, Transporte, Transporte Terrestre.

\begin{abstract}
Overview. Ground transportation is that transfer that takes place on the surface of the Earth, the vast majority are on wheels, it has its origin in the transfer of the human being, who, by the need to move or transfer their loads, according to weight or distance
\end{abstract}


He saw the need to improve this mechanism, from the rivers to the Mainland. The passage of time caused the appearance of the wheel in the future of engine, who was also immersed in its evolution and improvement, first use the fire, water, steam and then see the internal combustion engine, enhanced with the discovery of oil. Today the transportation points to new alternatives such as electricity, solar and artificial intelligence.

Keywords: Security, Transportation, Land Transport.

\section{Introducción}

El transporte, es la capacidad de desplazar personas o materiales de un espacio a otro, es el acto y consecuencia de trasladar algo de un lugar a otro. Para la real academia de la lengua española, permite nombrar también, a aquellos artilugios o vehículos que sirven para tal efecto, algunos ejemplos donde puede aparecer el término pueden ser: el transporte se demoró, el precio del transporte, necesito un transporte, etc.

El transporte en la actualidad es algo imprescindible para el despliegue de las actividades personales y de la sociedad en general, desde las actividades más simples hasta las que permiten la estabilidad y evolución de una nación, por ejemplo, el transporte forma parte de la logística, organización y servicio o una empresa, para garantizar la correcta distribución y comercialización de los productos al menor costo posible. En el plano personal, el transporte es parte de la vida cotidiana de cualquier ciudadano, atendiendo a que actividad realiza y cuan complejo se convierta el acceso a esta, por lo que debe hacer uso de él para su realización.

De manera general el transporte según: el medio se clasifica en aéreo, marítimo y terrestre, hablamos de transporte marítimo, cuando el traslado se realiza sobre el mar, este puede ser en barco, lancha, yate, velero, canoa, botes de remos o de motor. El 
transporte aéreo tiene como característica que el traslado se realiza por aire y este puede ser por avión, helicóptero, globos aerostáticos fundamentalmente, en el transporte terrestre el desplazamiento es por tierra, carreteras o raíles y los principales medios pueden ser: autobús, ferrocarril, coche, bicicleta, ciclomotor etc.

Desde la propiedad y uso del medio el transporte se clasifica en público o privado. El transporte público o transporte en común aplicado a todo transporte colectivo de pasajeros, donde los viajeros tienen que adaptarse a los horarios y a las rutas que ofrezca el operador, estos comparten no solo el medio de transporte disponibles, sino también en la mayoría a la ruta trazada, estos incluyen autobuses, taxis, trolebuses, tranvías, trenes, ferrocarriles suburbanos, ferris, avión y muchos otros. Una de las características que distingue al transporte público es que existe una tarifa que el usuario debe abonar para el uso del medio, también puede ser por tarjeta, las que usamos al subir al vehículo, la tarifa es descontada al introducir o presentar la tarjeta en la máquina lectora.

El transporte privado como su nombre lo dice se utiliza para referirse a los servicios de transporte que no están abiertos o disponibles para el público en general, en ellos se llevan a cabo tanto en el marco de la necesidad como en el de la actividad a realizar, existe la pertenencia del medio, y el propietario define el objetivo de su uso, la ruta y el tiempo para la realización de la actividad.

Adentrarse en la interpretación del transporte facilita entender la historia y comprender que la evolución del transporte esta aparejada a la historia de la humanidad, hace unos 5.000 años se suscitaron tres inventos importantes, estos fueron la rueda, la vela y el arado, los que colaboraron con la transformación de la humanidad, proporcionando cambios radicales en la vida del hombre, su repercusión influyo en áreas como, la agricultura, la alfarería, la cerámica, la navegación y el transporte, posibilitando la aceleración del movimiento de las personas y mercancías, los cultivos 
se ven beneficiados en tanto se implementa mayor productividad y rapidez en la realización de sus acciones, el surgimiento de la comunicación se extiende al acceso entre países, con el aprovechamiento de la fuerza del viento y aumentó el tamaño de las embarcaciones, promoviéndose así el establecimiento las de relaciones comerciales.

Pero se convierte en una necesidad al interior de cada país la necesidad de trasladar a las personas, los objetos y las mercancías, para ser distribuidos por diferentes sitios y territorios, es la solución de esta necesidad que da origen al transporte terrestre.

\section{Evolución del Transporte}

Para el surgimiento y evolución del transporte fue antecedida por la aparición de la rueda, la más antigua de la que se tiene noticias versa de Mesopotamia, donde aparecen las primeras imágenes de carros con ruedas, encontradas en las estepas de Eurasia; se piensa, que estas han sugerido debido al inmenso desafío de la evolución de la humanidad, que la invención de la rueda probablemente ocurrió una sola vez y se extendió desde su lugar de origen a otras partes del mundo, aunque otros creen que se desarrolló al mismo tiempo en diversas partes del mundo, por ejemplo, existe la versión de la rueda de los pantanos de Ljubljana, una rueda de madera que se encontró en la capital de Eslovenia en 2002 y que databa de 3150 a.C.

La rueda no era una invención perfecta. Por ejemplo, los camellos eran una forma de transporte mucho más eficiente en el ambiente desértico en comparación con la rueda entre los siglos II y VI a.D., en el Medio Oriente y África del Norte.

En la época precolombina (comprendida como la aparición de los primeros pobladores americanos, que se extiende hasta el 1492, con la conquista por los europeos con la colonización de Colón), donde ya en la cultura Incas, poseían un sistema de 
caminos interconectados a través de todo su imperio para trasladar diferentes tipos de mercancías y hacían uso de rústicos medios de transporte.

La evolución de la historia evidencia una rica transformación de los diferentes medios y usos del trasporte, desde los carros y diligencias tirados por caballos hasta los medios más sofisticados de la actualidad, gracias al aumento de tamaño y densidad de las poblaciones en las ciudades de las primeras civilizaciones, la necesidad de comunicación con otras regiones, aparejado a ello se convierte en necesario, el surgimiento de las carreteras, para el traslado de los medios de transporte que van surgiendo, esto como uno de los primeros signos, de una civilización avanzada, y así hacer llegar así suministros alimenticios allí donde hacía falta.

Los mesopotámicos fueron uno de los primeros constructores de carreteras hacia el año 3500 a.C. Le siguieron los chinos, los cuales desarrollaron un sistema de carreteras en torno al siglo XI a.C. y construyeron la Ruta de la Seda (la más larga del mundo) durante 2.000 años; Los incas de Sudamérica construyeron una avanzada red de caminos que no se consideran estrictamente carreteras. Las llamadas carreteras recorrían todos los Andes e incluían galerías cortadas en rocas sólidas (Revistas Arquys, 2012).

La bicicleta, que es un vehículo rápido, cómodo y económico, representa el medio de transporte más popular, su origen tiene base en el siglo XV, cuando Leonardo da Vinci diseña la primera bicicleta de la esta tenía las ruedas iguales, un asiento y pedales, la tracción se dirigía mediante una polea a la rueda trasera, pero carecía de dirección y la rueda delantera no podía moverse, así que no era práctica y se quedó en un esquema en los papeles del genio italiano llamada pomposamente "caballo de acero", para los niños esta constituye un juguete extraordinario, mientras que para los trabajadores les facilita a menudo su traslado. 
Su aparición está comprendida en Francia, en el año 1790, con la aparición del celerífero, al cual podríamos considerar como el antepasado de la bicicleta. Inventada por Sivrac. Aunque, algunos afirman que ya un siglo antes se conocía su uso. Y sostienen que un profesor de la Sorbona, llamado Ozanam, habría sido quien aplicó el principio de la bicicleta, en 1693, y logró construir una máquina similar muy elemental.

Esta es llamada “celerífero", tenía dos ruedas, una detrás de la otra, unidas por medio de una viga se montaba como a caballo, para avanzar con suficiente rapidez, el ciclista tenía que utilizar sus piernas y sus pies como aparatos de propulsión. Veinte años más tarde, un agrimensor alemán llamado Federico Drais, intentó perfeccionar la máquina de Sivrac y ajustó dos medas a un caballete de madera provisto de un gorrón (extremo rebajado de una madera o eje, que entra en un hueco de otro), puso una especie de manillar y agregó un asiento y la llamó la draisiana.

Su evolución continuó y se atribuye al francés Michaux o al alemán Fischer de Schweinfurt, en 1855, haber perfeccionado la máquina, con la introducción de la novedad de un doble pedal, dispuesto sobre la rueda delantera, lo que permitía convertir la propulsión por medio de los pies, en un movimiento continuo impulsado por los pedales, bajo el nombre de velocípedo ya que adquiría mayor velocidad.

En los años siguientes en busca de aumentar cada vez más la velocidad de estos aparatos, los mecánicos agrandaron la rueda delantera, que era la principal, y redujeron paulatinamente la rueda trasera, además se logró otro importante avance, que consistió en revestir con un anillo de caucho la llanta hueca de las ruedas; con ello, el vehículo se tomó más liviano. Estos aparatos de locomoción, fueron llamados biciclós.

Con la creación de los neumáticos, obra de un joven llamado Dunlop, hijo de un veterinario inglés, apasionado del ciclismo, quien trató de eliminar las sacudidas producidas por el vehículo e ideó un sistema que consistía en rodear las ruedas con 
pequeñas almohadillas llenas de aire, después de muchos experimentos llegó a obtener un tubo de caucho, delgado y flexible, que podía llenarse de aire comprimido y colocarse alrededor de las ruedas, con ayuda de su padre, en 1890 fabricó los primeros neumáticos. La bicicleta evolucionó a la motocicleta, al incorporar una caldera de vapor en la parte trasera, suprimiendo elementos como los pedales, mejorando el tipo de motor, hasta llegar a la moto conocida el día de hoy.

Comprender la evolución del transporte terrestre nos pone a la mira de la evolución del motor y como el hombre comenzó, con el uso su propia energía muscular, desarrollada a través de mecanismos simples como la palanca y la polea, luego la obtención de la energía necesaria para realizar las labores más extenuantes consistió en la domesticación de los animales para la sustitución de la energía humana por el animal, la mejora progresiva acontece con la implementación de los aparejos que permitieron el incrementando la capacidad de tiro de los animales. Un paso importante ha sido el aprovechamiento de las energías potenciales de la naturaleza como la hidráulica, el fuego y la eólica, que precursoras de los motores actuales.

El francés Denis Papin (1690), tuvo la clarividencia de usar la condensación del vapor de agua como mecanismo para producir vacío en el interior del cilindro, proporcionando, así el fundamento para la construcción de la máquina de vapor de Newcomen. La idea de Papin fue poco practicable, ya que el cilindro tenía la triple función de caldera, condensador y mecanismo transmisor de potencia, este fue el primero en aplicar la creación de vacío por condensación de vapor a la obtención de potencia mecánica.

En 1698, tras los trabajos en las minas de estaño de Cornish fueron obstaculizados, por la presencia de agua, para extraer esta, Thomas Savery diseñó y construyó un motor sin pistón al que llamó “The Friend Miner's" (El Amigo del Minero), siendo esta 
precursora del motor de vapor en 1712 construido por Thomas Newcomen, expresado en el hecho de que, fue el único utilizado por el hombre durante aproximadamente 150 años.

No se detiene la evolución de los automóviles, donde el hombre, va buscando la manera de inventar la forma de transportase rápida y cómodamente. En 1882, se descubrió el petróleo y con su uso, poco a poco fueron surgiendo aparatos que empleaban este combustible como fuerza impulsora.

Con la Primera Guerra Mundial las necesidades de transporte se incrementaron, y así surgieron los autobuses, y se perfecciona la gran industria del motor, que incluye tan variados métodos de transporte como el ferrocarril, transporte urbano, metro o tren de alta velocidad. Su evolución ha sido fundamental para para el avance de la propia Humanidad.

La industrialización europea, o la Edad Moderna, siglo XVI que se distingue por un avance en el comercio, surgimiento de la banca, evolución de los métodos financieros, progreso técnico en la navegación, la impresión, la relojería, la industria textil, etc., seguido por un aumentado marcado del crecimiento demográfico.

Sin embargo, todos los factores anteriores, distintivos de la Revolución Industrial, no cobran prosperidad, sin el desarrollo de los transportes, encargados de llevar las mercancías producidas en las fábricas hasta los mercados donde se consumirían.

\section{Surgimiento de las Vías para el Transporte Terrestre}

A la par del avance y perfeccionamiento del transporte, las carreteras, cobran gran importancia, por ejemplo, las más antiguas fueron construidas por los romanos. alrededor del 312 a.C., y la vía Faminia hacia el 220 a.C. lo que pone al poder, del Imperio romano en la cumbre de las sociedades por poseer un sistema de carreteras de 
unos $80.000 \mathrm{~km}$, estas contaban con 29 calzadas que partían de la ciudad de Roma, y una red que cubría todas las provincias conquistadas.

En esta época toda persona tenía derecho a usar las calzadas, según la ley romana, pero los responsables de su mantenimiento eran los habitantes del distrito por el que pasaba. Este sistema era eficaz para mantener las calzadas en buen estado, con la ausencia de la autoridad central del Imperio romano durante la edad media (del siglo $\mathrm{X}$ al XV), el sistema de calzadas nacionales empezó a desaparecer. A mitad del siglo XVII, el gobierno francés construyó aproximadamente $24.000 \mathrm{~km}$ de carreteras.

El imperio Inca comenzó a extenderse en la región de Cuzco hasta la región sur de las montañas Andinas de América del Sur, Norte de Chile y Sur de Colombia, cerca del fin del siglo XIV, en este tiempo ya existía una vía real de $16,000 \mathrm{~km}$, que unía Quito con Santiago de Chile, el tramo del norte, la Carretera de Alaska, fue construido en 1942, durante la Segunda Guerra Mundial, por razones militares.

En Costa Rica, la Panamericana alcanza su punto más alto en el Cerro Buena Vista $(3,505 \mathrm{~km})$, En la actualidad la carretera Panamericana, también llamada ruta Panamericana, es la carretera más larga del mundo, es un sistema colectivo de carreteras, de aproximadamente $25,800 \mathrm{~km}$ de largo, esta fue concebida en la $\mathrm{V}$ Conferencia Internacional de los Estados Americanos en 1923, enlaza casi a todos los países del hemisferio occidental del continente americano, se extiende desde Alaska en Norteamérica hasta la Isla de Chiloé en Chile y Patagonia argentina en Sudamérica.

Existe en esta un tramo importante, que impide que la carretera se conecte completamente, de unos $87 \mathrm{~km}$ de selva montañosa dura, que se ubica entre el extremo este de Panamá $(58 \mathrm{~km})$ y el noroeste de Colombia $(29 \mathrm{~km})$ llamado el Tapón de Darién. La carretera Panamericana se corta en Yaviza, (Panamá), y en Lomas Aisladas, (Colombia) aquí se retoma la Carretera panamericana en América del Sur. 
Desde la perspectiva anterior se amplía no solo la relación del trasporte con uso comercial y tecnológico, sino que aparece una nueva arista que es su empleo para el turismo, desde el siglo XIX con la aparición de los ferrocarriles como medio de transporte fundamentalmente en Europa y Estados Unidos, estos eran utilizados para asistir a los museos y visitar los monumentos por razones de entretenimiento, recreación.

Con el surgimiento de los primeros vehículos de combustión interna como medio de desplazamiento, se convierte con el pasar del tiempo, en el transporte una necesidad inseparable del turismo, lo mismo para aquel que tiene rutas y horarios establecidos, así como para el que es planificado de forma personal, familiar, grupal etc.

Entonces el transporte terrestre cobra gran importancia, ha de asegurarse que, ningún país puede avanzar si no tiene un transporte terrestre eficiente, esto incluye la técnica (entendida como los medios de transporte), su mantenimiento, abastecimiento y las carreteras, por lo que se realiza un gran esfuerzo para mejorar este aspecto en la sociedad, ya que conquistas sociales no serían lo mismo sin la calidad, la puntualidad y la seguridad del transporte que en ocasiones responde al sector público y en otras al privado dependiendo de a quien pertenezca la producción o empresa, pero siempre en beneficio de los consumidores.

\section{La Seguridad en la Transportación}

Actualmente, tanto para el transporte terrestre nacional como el internacional, debe optimizarse al máximo para hacer que sea seguro, ecológico y rápido, todo ello sin olvidar su fiabilidad, lo que genera que transportarse se convierta no solo en una necesidad sino también que su uso genere placer y seguridad. 
El aumento demográfico, provoca una mayor intensidad y frecuencia de desplazamientos esto agrava los efectos en el entorno por las emisiones de gases de efecto invernadero, sintetizando el verdadero problema de actualidad, en los espacios donde se concentra mayor población, el impacto del transporte no sólo afecta al medio ambiente sino también a la salud de la ciudadanía por la contaminación atmosférica.

El ruido es otro de los impactos que repercute directamente en la calidad de vida de la ciudadanía, a pesar de que en las últimas décadas las poblaciones se han acostumbrado al ruido originado por el tráfico.

La proyección de la seguridad en el transporte terrestre parte de conseguir indiscutibles puntos que ayudarán a que este se confirme, como efectivo y eficaz. Desde aquí que para, sea necesario que tanto las empresas como los particulares se centren en aspectos que garanticen elevar de forma sistemática el uso de los vehículos las carreteras y la interacción entre todos de forma gradual y creciente, un aspecto a tener en cuenta en perspectivas futuristas es la de la optimización de las rutas, para reducir el tiempo de transporte, y abaratar costos tanto en gasolina, como en mano de obra, esto se le imputan a la flota de camiones encargado de la distribución de producciones de diferentes empresas esto repercute en un impacto positivo en la bajada del precio del producto final.

Un aspecto que promete calidad en la transportación de producciones y de las personas, se centra en cuidar del chofer, que este tenga suficiente descanso antes de conducir, se alimente bien, se mantenga ágil, no viole las reglas de las horas de servicio y de descanso, cuide de su salud, y un aspecto relevante es que cuando el traslado se realice en horas nocturnas o prolongadas se mantenga un adecuado contacto con los conductores, de manera continuada, esta puede ayudar a reducir los accidentes en las 
carreteras, por otra parte, se debe asegurar que los trabajadores reciban la formación sobre medidas de seguridad a tener en cuenta.

La gestión de la seguridad del transporte terrestre implica también, equipar las flotas de camiones o buses, tanto público como privados con las medidas de seguridad y hacer que todos y cada uno sean sometidos a revisiones periódicas, que, todas las decisiones sobre seguridad sean tomadas y se exija su cumplimiento, estas son paradas, velocidad máxima permitida, revisiones de los medios de transporte, revisiones médicas, respeto se las señalizaciones.

La seguridad, especialmente la relacionada con la protección de los usuarios del servicio, la población y el medio ambiente, constituyen prioridad esencial de la actividad del sector y del sistema de transporte. En tal sentido, el transporte debe ofrecerse en condiciones que garanticen la movilización segura de las personas y las mercancías a su lugar de destino (Orjuela López, 2014, p.4).

En relación con el vehículo es significativo que sea inspeccionado antes de cada viaje, fundamentalmente los frenos, la batería, el combustible, los neumáticos, la dirección, la suspensión y el rodamiento, esto garantiza la seguridad vial desde el transporte.

Es necesario que durante el traslado se tengan en cuenta las siguientes medidas que garantizan la evitación de sucesos nefastos, como: mantener la distancia entre vehículos, dejando siempre suficiente espacio entre un vehículo y el que le antecede. El ajústese el cinturón de seguridad, así como exigir que el resto de las personas lo tengan ajustado, se confirma que el cumplimiento de esta medida minimiza las heridas en una colisión y otros accidentes, impidiendo que el pasajero se golpee con los elementos duros del interior o contra las personas en la fila de asientos anterior, y que sea arrojado fuera del vehículo. 
Importante resulta trasmitir, que se debe manejar siempre a la defensiva, esto implica que el conductor evite a los conductores agresivos, y no se realice este acto de conducir como un acto agresivo, la necesidad de disminuir la velocidad en las zonas en construcción en las carreteras, se debe promover que los conductores estén atentos a ayudar a y recibir ayuda durante el uso de las carreteras o de los transportes.

Otro aspecto a tomar en cuenta en términos de seguridad es el referido a la seguridad de las cadenas logísticas terrestres, por cuanto los países que tienen un mejor desempeño en seguridad son también más competitivos (Pérez Salas, 2013).

La cuestión estriba en que un bajo desempeño en términos de seguridad les resta potencial competitivo al país, la relación entre seguridad del transporte terrestre y competitividad, es compleja y demanda soluciones integradas y coordinadas nacional y regionalmente.

En el Ecuador los robos se registran principalmente a productos contenedorizados de importación en las inmediaciones del Puerto de Guayaquil, particularmente en las vías Perimetral y Naranjal cercanas al puerto donde los camioneros son intimidados por delincuentes que se movilizan en vehículos. Respecto a los productos, corresponden a aquellos que han sufrido incremento en los aranceles y por tanto, de fácil reducción (CLS News, 2009b, citado por Pérez Salas, 2013).

La seguridad del transporte terrestre está zanjada, además, por la higiene de los vehículos, particulares y de uso colectivo, estas normas se refieren fundamentalmente a las obligaciones a cumplir por los usuarios de estos medios que entre otras cuentan con la prohibición de: fumar en el interior del vehículo, producir ruidos innecesarios, conversar con los conductores, salvo asuntos estrictamente indispensables del servicio, arrojar en el vehículo papeles, cáscaras o cualquier objeto inservible, escupir, comer o 
beber en los vehículos, y cuanto pueda perturbar el decoro de un recinto público de acuerdo con el buen espíritu cívico.

Para el conductor es de estricto cumplimiento mantener y propiciar la higiene y seguridad de los pasajeros, los vehículos dispondrán de papelera para el depósito de los desperdicios, y estarán atentos a las necesidades de los usuarios.

\section{La transportación en la actualidad}

Es comprensible que la evolución de los medios de transporte terrestre, como parte de la estructura social no ha llegado a su máximo esplendor, que en la actualidad se abre a la tecnología una mirada futurista que solo era hasta hace poco producto de la ciencia ficción, pero que hoy podemos contar con novedosos vehículos y que la evolución apunta que estos sean más bellos, cómodos, y seguros, sirvan para combatir los atascos en las ciudades, y puedan desarrollar mayor velocidad, como ocurre con el uso del metro y del tranvía que circularán por encima o por debajo de las carreteras convencionales, además serán capaz de llevar en su interior a más de 1.000 personas.

Hoy es sólo un proyecto, que en la ciudad de Shanghái se quiere poner ya en marcha como pionera en el transporte moderno, el funcionamiento de un tren de levitación, "El Maglev", que va a más de 400 kilómetros por hora y que unirá al aeropuerto con el centro de la población. "El Transit" gigantesco autobús que va montado sobre raíles, cuenta con una longitud de 21,9 metros, 4,8 metros de anchura y deja, al menos, 2,1 metros de espacio libre al suelo, por donde va sobrepasando a los vehículos. Su velocidad máxima de 60 kilómetros por horas, para ser usado en amplias vías de circulación.

Aspecto a desplegar como perspectiva del transporte terrestre es el aprovechamiento de la energía solar, los fabricantes de automóviles buscan soluciones para huir de la 
gasolina, y para ello van saliendo al mercado los vehículos híbridos, los eléctricos con la electricidad como única fuerza motriz, los propulsores a gas, ahora se da una nueva mirada; que da paso a más automóviles que no van a precisar de un surtidor o de un enchufe, se trata de un vehículo que va a aprovechar, para sus movimientos, la fuerza del Sol, un modelo, denominado "Sion”, responde a un proyecto de Tesla con Solarcity, que monta unos paneles solares en la estructura del vehículo.

Desde el 2017 se despliega el proyecto de los primeros vehículos sin conductor, estos están de prueba en diferentes escenarios de circulación. En ellos se utiliza la tecnología de inteligencia artificial, que cuenta con visión artificial, reconocimiento de voz y recorre las calles de algunos países como Rusia, Alemania, Estados Unidos, “El Hamn”, es autónomo tiene capacidad para 12 personas y recorre unos 200 kilómetros, creado por la empresa de Internet Yandex, rusa, en colaboración con el fabricante de camiones Kamaz, también ruso, el fabricante alemán Daimler y el centro ruso de investigación Nami.

\section{Conclusiones}

El análisis desarrollado, permite comprender que el siglo XXI es el escenario ideal para que se perfeccione el empleo del transporte terrestre, con una nueva mirada de la humanidad, que posibilita el vínculo estrecho entre ciencia, técnica y tecnología, todo ello permitirá, que este sea mas optimo, se realice en menos tiempo, sea empleado por un mayor número de personas y que además contribuya al cuidado del hombre y del medio ambiente, pero que su calidad dependa de las medidas de seguridad que se tengan en cuenta.

\section{Referencias Bibliográficas}


Camarena Luhrs M. (2014) Transporte terrestre, un tema regional estratégico del Tratado de Libre Comercio de América del Norte. Revista Norteamérica, Año 9, número 2, julio-diciembre de 2014.

Camarena Luhrs M. (2014) 2013 “El Tratado de Libre Comercio: endeudamiento y costo soberano en México”, Ola financiera no. 14, enero-abril, pp. 37-59, Disponible en <http://www.olafinanciera. unam.mx/new_web/14/pdfs/Camarena-Olafinanciera14.pdf>.

Comisión Europea (2011) Libro Blanco del transporte. Hoja de ruta Hacia un espacio único europeo de transporte: por una política de transportes competitiva y sostenible. Luxemburgo: Oficina de Publicaciones de la Unión Europea, ISBN 978-92-79-18274-7 doi:10.2832/42444

Heywood J.B., “Internal Combustion Engine Fundamentals”, McGraw-Hill, New York, 1989.

De la Mora Sánchez, L. M. (2011) “Transporte terrestre: el gran pendiente del tlcan”, Fortuna, pp. 1-3.

Obert F. Edward, "Motores de Combustión Interna”, Cecsa, Mexico, 1997 <http://revistafortuna.com.mx/contenido/2011/01/10/transporte-terrestre-el-granpendiente-del-tlcan/>.

Orjuela Lopez S. (2014) Seguridad Logística, Un Aliado en las operaciones terrestres en transporte de carga. Tesis presentada para optar por la Especialización en Administración de la Seguridad. Curso 45 - Nivel II. Bogotá - Colombia 2014.

Pérez Salas (2013) Seguridad de la cadena logística terrestre en América Latina. Publicación de las Naciones Unidas ISSN 1680-9017, LC/L.3604. Santiago de Chile. 
Revista ARQHYS. 2012, 12. Historia de las carreteras. Equipo de colaboradores y profesionales de la revista ARQHYS.com. Obtenido 02, 2018, de http://www.arqhys.com/contenidos/carreteras-historia.html.

Fuente: http://www.arqhys.com/contenidos/carreteras-historia.html 